\title{
The Number of Commodities Required to Represent a Market Game*
}

\author{
SERgiu Hart \\ Department of Statistics, Tel-Aviv University, \\ Tel-Aviv, Israel
}

Received June 25, 1980; revised October 27, 1980

An $n$-person game with side payments that can arise from an economic market is called a market game. It is proved here that any $n$-person market game can be obtained from a market with at most $n-1$ commodities. Moreover, no smaller number will suffice in some cases (e.g., the unanimity game). This result is then applied to settle a question regarding market games with a continuum of traders. Journal of Economic Literature Classification Numbers: 021, 022.

The following problem is studied here: What is the minimal number of commodities required to represent a totally balanced $n$-player game (with side payments) ${ }^{1}$ Shapley and Shubik [4] showed that every such game is a market game-i.e., it can be derived from a "market"; their construction (of the "direct market") requires $n$ commodities. It is proved here that $n-1$ suffice (Proposition 2); moreover, this bound is exact-there are $n$-player market games for which $n-1$ goods are necessary (Proposition 1). This last result is then applied to non-atomic games (where the original question - setlled here as Proposition 3-was raised).

The $n$-player unanimity game is defined as follows: $v(S)=0$ for all $S \varsubsetneqq N$ and $v(N)=1$, where $N=\{1,2, \ldots, n\}$. It is clearly a totally balanced game; a market which generates it, is for example, ${ }^{2}\left(N, \mathbb{R}_{+}^{n-1}, A, U\right)$, where, for $i \neq n$,

* This research was supported by the Institute for Advanced Studies, the Hebrew University, Jerusalem, Israel, and by National Science Foundation Grant SOC75-21820-A01 at the Institute for Mathematical Studies in the Social Sciences, Stanford University. The author wishes to thank R. J. Aumann, J. F. Mertens and L. S. Shapley for very helpful discussions.

'For the non-side-payments case, where the problems are not yet completely settled, see [6], which includes an overview and references.

${ }^{2}$ The notations of Shapley and Shubik [4] are followed here. $N$ is the set of agents, $\mathbb{R}_{+}^{m}$ the commodity space, $A=\left\{a^{\prime}\right\}_{i \in N}$ the initial endowments and $U=\left\{u^{\prime}\right\}_{i \in N}$ continuous concave utility functions (not necessarily non-decreasing). The market game $v$ is defined by $v(S)=$ $\max \left\{\sum_{i \in S} u^{l}\left(x^{i}\right) \mid \sum_{i \in S} x^{l}=\sum_{i \in S} a^{l}\right.$, all $\left.x^{l} \in \mathbb{R}_{+}^{m}\right\}$ for all $S \subset N$. 
$a^{i}$ is ${ }^{3}$ the $i$ th unit vector in $\mathbb{R}^{n-1}$ and $u^{i} \equiv 0$; for $i=n, a^{n}=0$ and $u^{n}\left(x_{1}, x_{2}, \ldots, x_{n-1}\right)=\min \left\{x_{1}, x_{2}, \ldots, x_{n-1}\right\}$.

Proposition 1. Let $\left(N, \mathbb{R}_{+}^{m}, A, U\right)$ be a market generating the unanimity game $v$ on $N$. Then $m \geqslant n-1$.

Proof. Since $v(\{i\})=0$, we have $u^{i}\left(a^{i}\right)=0$; since $v(N \backslash\{i\})=0$, we have

$$
0=\max \left\{\sum_{j \neq i} u^{j}\left(x^{j}\right) \mid \sum_{j \neq i} x^{j}=\sum_{j \neq i} a^{j}, \text { all } x^{j} \in \mathbb{R}_{+}^{m}\right\},
$$

hence the maximum is attained at $x^{j}=a^{j}$ for all $j \neq i\left(\sum_{j \neq i} u^{j}\left(a^{j}\right)=\right.$ $\left.\sum_{j \neq i} 0=0\right)$.

Now $v(N)=1$, hence there is an allocation $\left(y^{l}\right)_{i \in N}$ such that

$$
\sum_{i \in N} y^{i}=\sum_{i \in N} a^{i}, \quad \sum_{i \in N} u^{i}\left(y^{\prime}\right)=1 .
$$

Let us define now new concave functions $\hat{u}^{i}: \mathbb{R}_{+}^{m} \rightarrow(-\infty,+\infty)$ as follows:

$$
\begin{array}{rlrl}
\hat{u}^{\prime}(x) & =t u^{i}\left(a^{i}\right)+(1-t) u^{i}\left(y^{i}\right), & & \text { for } x=t a^{i}+(1-t) y^{i}, \\
& =-\infty, & & \text { with } 0 \leqslant t \leqslant 1, \\
& & \text { otherwise. }
\end{array}
$$

Then $\hat{u}^{i}$ are polyhedral concave functions (cf. Rockafellar [3, pp. 172-173]), and $\hat{u}^{i} \leqslant u^{i}$. Let $\hat{v}$ be the market game generated by $\left(N, \mathbb{R}_{+}^{m}, A, \hat{U}\right)$, then $\hat{v} \leqslant v$; however, since $\hat{u}^{i}\left(a^{t}\right)=u^{i}\left(a^{l}\right)$ and $\hat{u}^{i}\left(y^{l}\right)=u^{i}\left(y^{i}\right)$, we actually have $\hat{v}=v$.

Now consider the functions $\hat{u}_{s}$, defined for $S \subset N$, as follows:

$$
\hat{u}_{S}(x)=\sup \left\{\sum_{i \in S} \hat{u}^{l}\left(x^{i}\right) \mid \sum_{i \in S} x^{i}=x, \text { all } x^{i} \in \mathbb{R}_{+}^{m}\right\} .
$$

Then $\hat{u}_{s}$ are polyhedral concave functions (cf. Rockafellar $\mid 3$, Corollary 19.3.4]). In particular, for each $i \in N$, the function $\hat{u}_{N \backslash(i)}$ is subdifferentiable at $\sum_{j \in N \backslash(i)} a^{j}$ (cf. Rockafellar [3, Theorem 23.10]); i.e., there is $p^{i} \in \mathbb{R}^{m}$ such that

$$
p^{\prime} \in \partial \hat{u}_{N \backslash(i)}\left(\sum_{J \in N \backslash(I)} a^{j}\right)
$$

\footnotetext{
${ }^{3}$ Subscripts will be used for coordinates, and superscripts for players.
} 
But this clearly implies $p^{i} \in \partial \hat{u}^{k}\left(a^{k}\right)$ for all $k \neq i$; indeed,

$$
\begin{aligned}
\hat{u}^{k}(x) & =\sum_{j \neq i, k} \hat{u}^{j}\left(a^{j}\right)+\hat{u}^{k}(x) \leqslant \hat{u}_{N \backslash i)}\left(\sum_{j \neq i, k} a^{j}+x\right) \\
& \leqslant \hat{u}_{N \backslash(i)}\left(\sum_{j \neq i} a^{j}\right)+p^{i} \cdot\left(\sum_{j \neq i, k} a^{j}+x-\sum_{j \neq i} a^{j}\right) \\
& =\hat{u}^{k}\left(a^{k}\right)+p^{i} \cdot\left(x-a^{k}\right) .
\end{aligned}
$$

Hence $\left\{\partial \hat{u}^{k}\left(a^{k}\right)\right\}_{k=1}^{n}$ is a collection of $n$ convex sets in $\mathbb{R}^{m}$, such that the intersection of every $n-1$ of them is non-empty; if $m \leqslant n-2$, Helly's theorem (Rockafellar [3, Theorem 21.6]) would imply the existence of a vector $p$ with $p \in \partial \hat{u}^{k}\left(a^{k}\right)$ for all $k \in N$. Then

$$
1=\sum_{k=1}^{n} \hat{u}^{k}\left(y^{k}\right) \leqslant \sum_{k=1}^{n}\left[\hat{u}^{k}\left(a^{k}\right)+p \cdot\left(y^{k}-a^{k}\right)\right]=0,
$$

since $\hat{u}^{k}\left(a^{k}\right)=0$ and $\sum_{k} y^{k}=\sum_{k} a^{k}$. This contradiction shows that $m \geqslant$ $n-1$.

Remark. Note that each $u^{i}$ could be just a concave function with values in $[-\infty,+\infty)$ (i.e., not necessarily finite on all $\mathbb{R}_{+}^{m}$ ).

Proposition 2. Let $(N, v)$ be a totally balanced game. Then $v$ can be derived from a market with $n-1$ goods. Moreover, the set of competitive payoff vectors of this market and the core of $v$ coincide.

Remark. In Shapley and Shubik [5], it is shown that the competitive vectors of the direct market-with $n$ goods-enjoy the same property.

Proof. Consider the restriction of $v$ to $N^{\prime}=\{1,2, \ldots, n-1\}$. It is a totally balanced game of $n-1$ players; let $\left(N^{\prime}, \mathbb{R}^{n-1}, A^{\prime}, U^{\prime}\right)$ be the corresponding direct market. Define $a^{n}=0$ and $u^{n}: \mathbb{R}_{+}^{n-1} \rightarrow \mathbb{R}$ by

$$
u^{n}(x)=\max \left\{\sum_{s \subset N} \gamma_{s} v(S) \mid \sum_{s \subset N} \gamma_{S} e^{s}=(x, 1), \text { all } \gamma_{s} \geqslant 0\right\}
$$

where $(x, 1)=\left(x_{1}, x_{2}, \ldots, x_{n-1}, 1\right) \in \mathbb{R}^{n}$, and $e^{s} \in \mathbb{R}_{+}^{n}$ is the characteristic vector of $S$ (i.e., $e_{i}^{S}=1$ or 0 according to $i \in S$ or $i \notin S$ ). It is easy to check that $u^{n}$ is a continuous concave function. Denote by $\hat{v}$ the market game generated by $\left(N, \mathbb{R}_{+}^{n-1}, A, U\right)$.

We show now that $\hat{v}=v$. For $S \subset N^{\prime}$, it is clear that $\hat{v}(S)=v(S)$. When $n \in S$, we have

$$
\hat{v}(S) \geqslant u^{n}\left(\sum_{i \in S} a^{l}\right)+\sum_{i \in S^{\prime}} u^{i}(0)
$$


where $S^{\prime}=S \backslash\{n\}$. Now $u^{i}(0)=0$ and $\left(\sum_{i \in S} a^{i}, 1\right)=e^{s}$; therefore, choosing $\gamma_{S}=1$ and $\gamma_{T}=0$ for $T \neq S$ in the definition of $u^{n}\left(\sum_{i \in S} a^{i}\right)$, we obtain $\hat{v}(S) \geqslant v(S)$. Conversely, let $\left(x^{i}\right)_{i \in S}$ be an $S$-allocation (i.e., $\sum_{i \in S} x^{i}=$ $\sum_{i \in S} a^{i}$ ). Then, for each $i \in S^{\prime}$, we obtain $\gamma_{T}^{i} \geqslant 0$ for all $T \subset N^{\prime}$ such that

$$
\begin{aligned}
u^{i}\left(x^{i}\right) & =\sum_{T \subset N^{\prime}} \gamma_{T}^{i} v(T), \\
\sum_{T \subset N^{\prime}} \gamma_{T}^{i} e^{T} & =\left(x^{i}, 0\right)
\end{aligned}
$$

(see Shapley and Shubik [4, Eqs. (4-2) and (4-3)]). For $i=n$, we have $\gamma_{T}^{n} \geqslant 0$ for all $T \subset N$ such that

$$
\begin{aligned}
u^{n}\left(x^{n}\right) & =\sum_{T \subset N} \gamma_{T}^{n} v(T), \\
\sum_{T \subset N} \gamma_{T}^{n} e^{T} & =\left(x^{n}, 1\right) .
\end{aligned}
$$

Now let $\gamma_{T}=\sum_{i \in S} \gamma_{T}^{i}$ for $T \subset N^{\prime}$, and $\gamma_{T}=\gamma_{T}^{n}$ for $T \ni n$; then

$$
\sum_{T \in N} \gamma_{T} e^{T}=\left(\sum_{i \in S} x^{i}, 1\right)=\left(\sum_{i \in S} a^{i}, 1\right)=e^{S}
$$

which implies

$$
\sum_{T \subset N} \gamma_{T} v(T) \leqslant v(S)
$$

since $v$ is totally balanced. Hence

$$
\sum_{i \in S} u^{i}\left(x^{i}\right)=\sum_{T<N} \gamma_{T} v(T) \leqslant v(S),
$$

from which it follows that $\hat{v}(S) \leqslant v(S)$. This ${ }^{4}$ completes the proof that the above market indeed generates $v$.

We come now to the second part. We have to show that if $\beta=$ $\left(\beta^{1}, \beta^{2}, \ldots, \beta^{n}\right)$ belongs to the core of $v$, then it is a competitive payoff vector of our market (the other direction is well known). Denote $\pi=$ $\left(\beta^{1}, \beta^{2}, \ldots, \beta^{n-1}\right) \in \mathbb{R}^{n-1}, \quad x^{l}=(0,0, \ldots, 0) \in \mathbb{R}^{n-1}$ for $i \neq n$ and $x^{n}=$ $(1,1, \ldots, 1) \in \mathbb{R}^{n-1}$. We will show that $(\pi, x)$ is a competitive equilibrium, and that the corresponding payoff vector is precisely $\beta$.

\footnotetext{
${ }_{4}^{4}$ The reason the above construction need not work if we do not use the direct market for $N^{\prime}$, is that the vectors $\sum_{t \in S} a^{\prime}$ for $S \subset N^{\prime}$ may be linearly dependent and the function $u^{n}$ cannot be defined.
} 
Indeed, $\sum_{i=1}^{n-1} \pi_{i}=\sum_{i \in N^{\prime}} \beta^{i} \geqslant v\left(N^{\prime}\right)$; hence, for all $i \in N^{\prime}$, we have $u^{i}(x) \leqslant$ $\pi \cdot x$ for all $x \in \mathbb{R}_{+}^{n-1}$ (the same proof as Shapley and Shubik [5, Eq. (16)]). Therefore

$$
\beta^{i}=u^{i}(0)-\pi \cdot\left(0-a^{i}\right) \geqslant u^{i}(x)-\pi \cdot\left(x-a^{i}\right) .
$$

As for $i=n$, note that

$$
u^{n}\left(x^{n}\right)=v(N)
$$

(see the proof above that $v(S)=\hat{v}(S)$, for $S=N$ ); hence

$$
u^{n}\left(x^{n}\right)-\pi \cdot\left(x^{n}-a^{n}\right)=v(N)-\sum_{i \in N^{\prime}} \beta^{i}=\beta^{n} .
$$

Let $x \in \mathbb{R}_{+}^{n-1}$, then

$$
\sum_{S \subset N} \gamma_{S} e^{s}=(x, 1)
$$

implies, since $\beta \in$ core $v$, that

$$
\sum_{S \subset N} \gamma_{S} v(S) \leqslant \sum_{S \subset N} \gamma_{S}\left(e^{S} \cdot \beta\right)=(x, 1) \cdot \beta=\pi \cdot x+\beta^{n} ;
$$

therefore

$$
u^{n}(x)-\pi \cdot\left(x-a^{n}\right) \leqslant \beta^{n}=u^{n}\left(x^{n}\right)-\pi \cdot\left(x^{n}-a^{n}\right),
$$

completing the proof.

Remark. The idea behind the above representation of $v$ is that the $n$th player does not "bring" to the market any goods-he contributes, instead, his "good" utility function (or, production function-depending on the interpretation). This is possible because the utilities are transferable.

An $n$-player game $v$ can be regarded as a (real) function defined on the vertices of the unit $n$-cube (by identifying $S \subset N$ with $e^{s} \in\{0,1\}^{n}$ ). Then $v$ is totally balanced if and only if it can be (uniquely) extended to a continuous, concave, and positively homogeneous of degree one function on the unit $n$ cube $^{5}[0,1]^{n}$ (or even, on all $\mathbb{R}_{+}^{n}$ ); see Shapley and Shubik ([4, Sect. 4; 5, Footnote 5]).

This suggests a natural candidate for the space of non-atomic market games. With the additional requirement of differentiable utilities (rather than just continuous), Aumann and Shapley [1, p. 240] consider the space $H$ of all games in $p N A$ (the space of "differentiable" non-atomic games) that are

"This is the so-called set of "fuzzy" or "ideal" subsets of $N$. 
concave $^{6}$ and positively homogeneous of degree one. $H_{+}$is then the subset of all monotone games in $H$.

It is known that $H_{+}$contains the differentiable non-atomic market games-see Aumann and Shapley [1, proof of Proposition 40.26]). We show here that the converse is false.

Proposition 3. There are non-atomic games in $H_{+}$that are not market games (i.e., cannot be generated by markets).

We prove first a preliminary result which is a direct consequence of Proposition 1. Let $(I, \mathscr{C})$ be a measure space, assumed to be isomorphic to the unit interval $[0,1]$ with its Borel $\sigma$-field. Let $\mu_{1}, \mu_{2}, \ldots, \mu_{n}$ be $n$ mutually singular non-atomic probability measures on $(I, \mathscr{C})$, and define

$$
v=\sqrt[n]{\mu_{1} \cdot \mu_{2} \cdots \cdot \mu_{n}} .
$$

Then $v$ is a market game. For example, it can be generated using $n$ commodities as follows: let $T_{1}, T_{2}, \ldots, T_{n}$ be a partition of $I$ such that $T_{l}$ is a support of $\mu_{i}$; the initial endowment a $(t)$ of each $t \in T_{i}$ is the $i$ th unit vector $e^{t} \in \mathbb{R}_{+}^{n}$; the utility function of each $t \in I$ is $u(x)=n \cdot \sqrt[n]{x_{1} \cdot x_{2} \cdot \cdots \cdot x_{n}}$, and the population measure is $\mu=\left(\mu_{1}+\mu_{2}+\cdots+\mu_{n}\right) / n$. Actually, $n-1$ commodities suffice, as in Proposition 2.

Proposition 4. A non-atomic market generating the above $v$ has at least $n-1$ commodities.

Proof. Let $\left((I, \mathscr{C}, v), \mathbb{R}_{+}^{m},\{\mathbf{a}(t)\}_{t \in I},\left\{u_{t}\right\}_{t \in I}\right)$ be a market generating $v$. Let $T_{1}, T_{2}, \ldots, T_{n}$ be as above, and define, for $i=1,2, \ldots, n$,

$$
\begin{aligned}
a_{i} & =\int_{T_{l}} \mathbf{a} d v \\
u^{i}(x) & =\sup \left\{\int_{T_{i}} u_{t}(\mathbf{x}(t)) d v(t) \mid \int_{T_{i}} \mathbf{x} d v=x, \mathbf{x}(t) \in \mathbb{R}_{+}^{m} \text { for all } t\right\} .
\end{aligned}
$$

Then $u^{i}$ is a concave function (with possible values $-\infty$ ). Note that, if we replace each $T_{i}$ by a player $i$, we obtain the $n$-player unanimity game; moreover, $\left(N, \mathbb{R}_{+}^{m},\left\{a^{i}\right\}_{i \in N},\left\{u^{i}\right\}_{i \in N}\right)$ is an $n$-player market generating it. By Proposition 1 (see the remark following its proof), $m \geqslant n-1$.

Proof $^{7}$ of Proposition 3. Let $\left\{\mu_{n i}\right\}_{n=1,2, \ldots ; i=1,2, \ldots, n}$ be a (countably) infinite triangular array of mutually singular non-atomic probability

\footnotetext{
6 Their definition actually requires superadditivity instead of concavity; however, in the presence of homogeneity, the two concepts are equivalent.

${ }^{7}$ The idea of this proof is due to R. J. Aumann.
} 
measures on $(I, \mathscr{C})$ (for example, take the normalized restrictions of a nonatomic measure to a countable partition of $I$ into non-null sets). Define

$$
v=\sum_{n=1}^{\infty} 2^{-n} \sqrt[n]{\mu_{n 1} \cdot \mu_{n 2} \cdots \cdot \mu_{n n}}
$$

and let $v_{N}$ be the partial sum to $N$. Then $v_{N} \in p N A$ (by Theorem $\mathrm{C}$ in Aumann and Shapley [1, p. 25], $\sqrt[n]{\mu_{n i}} \in p N A$, which is an algebra), $v_{N}$ is positively homogeneous of degree 1 (see [1, Eq. (22.18)]), it is superadditive and monotone. Hence $v_{N} \in H_{+}$. Moreover, $v-v_{N}$ is also a monotone game, hence

$$
\left\|v-v_{N}\right\|=\left(v-v_{n}\right)(I)=\sum_{n=N+1}^{\infty} \frac{1}{2^{n}}=\frac{1}{2^{N}} \underset{N \rightarrow \infty}{\longrightarrow} 0,
$$

which, since $H_{+}$is closed, implies $v \in H_{+}$.

If $v$ is generated by a market, then, by considering the restriction to the support of $\left\{\mu_{n i}\right\}_{i=1}^{n}$, it must have at least $n-1$ goods (Proposition 3 ). This is true for all $n$, so no finite number of commodities suffice.

The importance of this last result lies in showing that, in order to study (differentiable) market games, one need not consider the whole space $H_{+}$; the finite dimensional "character" of the markets may be a useful property. A similar situation occurs in the non-differentiable case-cf. Hart [2, Open Problem A]. See also the forthcoming paper of Dubey and Neyman.

\section{REFERENCES}

1. R. J. Aumann and L. S. Shapley, "Values of Non-Atomic Games," Princeton Univ. Press, Princeton, N.J., 1974.

2. S. Hart, Measure-based values of market games, Math. Operations Res. 5 (1980), 197-228.

3. T. R. Rockafellar, "Convex Analysis," Princeton Univ. Press, Princeton, N.J., 1970.

4. L. S. Shapley ANd M. ShUbik, On market games, J. Econ. Theory 1 (1969), 9-25.

5. L. S. Shapley AND M. Shubik, Competitive outcomes in the cores of market games, Internat. J. Game Theory 4 (1975), 229-237.

6. R. J. Weber, Attainable sets and markets: An overview, in "Generalized Concavity in Optimization and Economics" (M. Avriel, S. Schaible and W. T. Ziemba, Eds.), Academic Press, New York, 1981. 